\title{
Serum Mannose Binding Lectin Levels in Early Onset Neonatal Sepsis
}

\author{
Andi Risko Amalia*, Ema Alasiry, Dasril Daud \\ Department of Pediatrics, Medical Faculty, Hasanuddin University, Makassar, South Sulawesi, Indonesia
}

Email address:

risko.amalia@gmail.com (A. R. Amalia), alasiryema@yahoo.com (E. Alasiry),drdasri@gmail.com (D. Daud)

To cite this article:

Andi Risko Amalia, Ema Alasiry, Dasril Daud. Serum Mannose Binding Lectin Levels in Early Onset Neonatal Sepsis. American Journal of Health Research. Vol. 3, No. 3, 2015, pp. 135-139. doi: 10.11648/j.ajhr.20150303.14

\begin{abstract}
Introduction: Early Onset Neonatal Sepsis (EONS) is still the most cause of neonatal morbidity and mortality. The diagnosis is difficult to establish because of nonspecific clinical features and laboratory findings. Mannose binding lectin (MBL) is one of the acute phase proteins that can show progressing infection process. Objective: To determine the serum MBL levels of neonates with EONS and not EONS. Methods: This study was conducted as a cross sectional study from September to November 2014. The population included 92 neonates baby whose mother has risk factors of sepsis and admitted to Dr. Wahidin Sudirohusodo Hospital and joined hospital. The subjects were divided into two groups, EONS and Not EONS group based on clinical features and laboratory findings. The MBL serum level was measured on each group. Results: Statistical analyses showed median value of serum MBL of EONS group $(0.88 \mu \mathrm{g} / \mathrm{mL})$ was significant lower compare with Not EONS group $(0.93 \mu \mathrm{g} / \mathrm{mL})$, with $\mathrm{p}=0.00(\mathrm{p}<0.05)$. The cutoff point of $\leq 0.93 \mu \mathrm{g} / \mathrm{mL}$ is the best levels to distinguish between EONS and Not EONS ( $\mathrm{p}=0.01$, sensitivity $76 \%$, specificity $56 \%$, positive predictive value $60 \%$, negative predictive value $67 \%$, COR 3.182, 95\%CI 1.306-7.752). Conclusion: MBL serum levels on EONS group were lower than Not EONS group.
\end{abstract}

Keywords: Mannose Binding Lectin, Early Onset Sepsis, Neonates

\section{Introduction}

It is estimated that about 3 million deaths or 40 percent of all deaths in children under 5 , occurred within the first 28 days of age. The mortality risk is highest in the newborn period then decline with the age in days, months and years. Neonatal sepsis is an important cause of morbidity and mortality in newborn. The incidence of neonatal sepsis varies from 1-4/1000 live births in developed countries to 10-50/ 1000 live births in developing countries. ${ }^{1}$ Sepsis occurs in less than $1 \%$ of newborn, but it is responsible for $30 \%$ deaths in newborn. Survival likelihood of infants with neonatal sepsis is very small, even for the infants under hospital care. The identification and treatment for neonatal sepsis and infection are still lack in many developing countries, because the sick newborn shows nonspecific symptoms and signs, it is still difficult to diagnose the neonatal sepsis even in hospitals with comprehensive facilities. ${ }^{2}$

In Indonesia, $37 \%$ of infant mortality is death of the newborn period up to 28 days of age, due to low birth weight, sepsis, asphyxia, respiratory distress disease, infectious disease, hypothermia, metabolic diseases and congenital defects; compared to other countries such as US, Europe, Russia, Australia, Arabian, Egypt, Malaysia and Thailand, the neonatal mortality rate in Indonesia is quite high (5-29 / 1000). ${ }^{3}$

Neonatal sepsis is a clinical syndrome caused by microorganism invasion into the bloodstream which occurs within the first month of life. Neonatal sepsis is divided into early onset sepsis (EONS) and late-onset sepsis (LONS). In early onset sepsis, the baby usually was exposed to microorganisms during the intra-partum period from the mother's genital organs. Other exposure to microorganisms is related to delivery process, through ruptured membrane, vaginal flora or other pathogens that rose and reach the amniotic fluid and the fetal. ${ }^{4}$

Immune response is the first defense line of body mechanism and it is important to prevent infection. Adaptive immune system in newborn is not fully developed yet, because B-cells and T-cells are still immature. ${ }^{5}$ Soon after the birth, newborns are exposed to various pathogens. Because the adaptive immunity's not present; therefore, natural immunity plays a very important role. Complement system is the key element in innate immunity, whose role is to eliminate the microorganism and to increase the adaptive 
immune response in the newborn period. Complement activation occur through classic pathway, alternative pathway and lectin pathway. One of the lectin pathway components is mannan binding lectin (MBL) which is a pattern recognition molecule that has a part similar to collagen. MBL function is to activate the complement system. ${ }^{6}$

Based on the study conducted by Ozkan H. et al., they concluded that a low MBL levels is a risk factor for neonatal sepsis. ${ }^{7}$ There's a controversy about MBL levels and the occurrence of sepsis. ${ }^{8,9}$ According to a study by Benedetti it was found that serum MBL levels in newborn with sepsis is $0.45 \mathrm{mcg} / \mathrm{ml}$, while in newborn without sepsis was 1.45 $\mathrm{mcg} / \mathrm{ml}^{8}{ }^{8}$ Levels of circulating $\mathrm{MBL}$ varies in highly suspected of sepsis. ${ }^{10}$

There's dilemma in sepsis management, the mortality rate will increase when the treatment is delayed, while on the other hand, it shall be over diagnosis occur due to atypical clinical features, the patients and the family would be at loss.
Besides, new information on sepsis pathogenesis and the course of disease within the last decade gives a new alternative to treat the neonatal sepsis in a more effectives and efficient approach.

Therefore, it is important to conduct a study to assess the serum MBL levels in neonatal sepsis. This study aims to analyze the serum MBL levels in EONS and not EONS.

\section{Materials and Methods}

This is an observational study using a cross sectional approach to evaluate the influence of serum mannose binding lectin levels on early onset neonatal sepsis. Newborn age up to 72 hours was consecutively recruited into this study. They are the patients' treated in Dr. Wahidin Sudirohusodo Hospital and other networking hospital in Makassar or the referral patients' outside Makassar, who met the criteria as samples from September until November 2014.

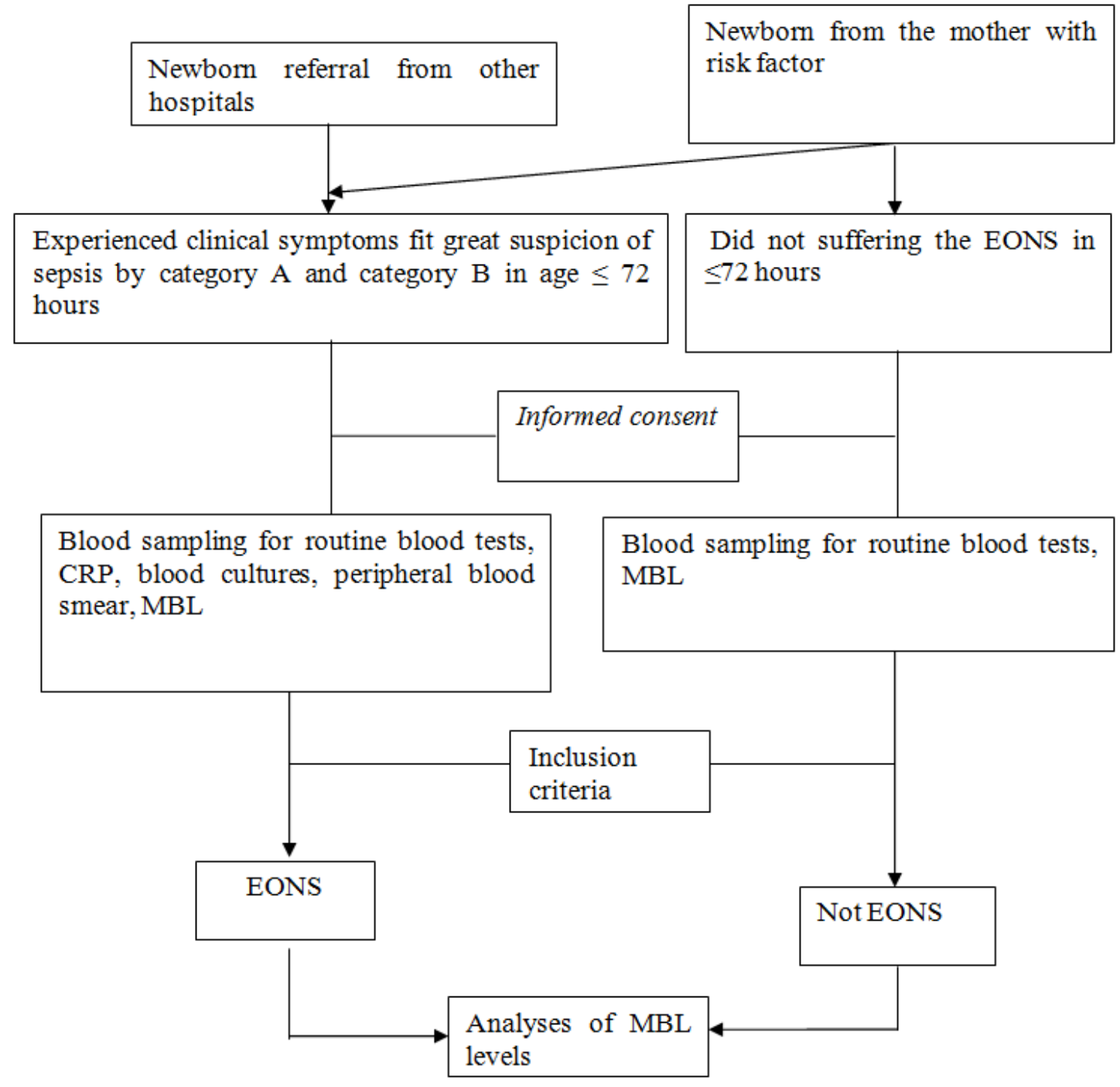

Figure 1. Study flow scheme.

The criteria for participation were divided into the inclusion and exclusion. The inclusion criteria are the newborn age $\leq 72$ hours with EONS, birth from the mother with risk factor but did not suffering the EONS in $\leq 72$ hours, 
single birth, and the blood samples are successfully obtained and stored. While, the exclusion criteria are the newborn obtained the antibiotics therapy before sampling, low birth weight, neonatal asphyxia, congenital abnormalities, birth from the mother with another disease (in addition the risk factors of sepsis), and lyses of blood samples. Then, the samples are divided into the group of EONS and not EONS.

This study was approved by the Ethics and Industry Research Committee of the hospital and Medical Faculty of Hasanuddin University. Written informed consent was obtained from the patients' parents or legal guardian following full and detail explanation regarding the study's protocol. Further analyses are performed with appropriate statistical methods by univariate and bivariate analyses.

\section{Result}

Table 1 shows the sample characteristics. From all the 92 samples, there are $42(45.7 \%)$ male and 50 (54.3\%) female. Fifty four samples $(58.7 \%)$ are from the maternal fever and the rest are 38 samples $(41.3 \%)$ of non fever. Samples from the mother with leucocytosis are $19(20.7 \%)$ patients, and $73(79.3 \%)$ are not from the mother with high leucocytes levels. Samples with history of premature rupture of membrane are $37(40.2 \%)$ infants and $55(59.8 \%)$ are not. There are 79 samples $(85.9 \%)$ with greenish amniotic fluid, and 13 samples $(14.1 \%)$ with clear amniotic fluid.

Table 1. Study sample characteristics.

\begin{tabular}{lll}
\hline No. & Sample Characteristics & Total $(\mathbf{n = 9 2}) \mathbf{n}(\mathbf{\%})$ \\
\hline 1. & Sex & \\
& Male & $42(45.7)$ \\
& Female & $50(54.3)$ \\
2. & Mother's temperature & \\
& Maternal Fever & $54(58.7)$ \\
& Non maternal fever & $38(41.3)$ \\
3. & Mother's leucocytes & $19(20.7)$ \\
& Leucocytosis & $73(79.3)$ \\
& No Leucocytosis & $37(40.2)$ \\
4. & Premature Rupture of Membrane (PROM) & $55(59.8)$ \\
& PROM & $79(85.9)$ \\
& Non PROM & $13(14.1)$ \\
\hline
\end{tabular}

The 92 study samples were grouped into EONS and not EONS group based on their history taking, physical examination and laboratory results. The EONS group consists of 46 samples; either the not EONS group. All samples in not EONS group were born in Dr. Wahidin Sudirohusodo hospital, while some of the samples in EONS group were referred from other networking hospitals in South Sulawesi, which then treated in Dr. Wahidin Sudirohusodo or at the networking hospitals. Of total 46 samples in EONS group, 22 samples are referral patients, and 24 samples are newborns in Dr. Wahidin Sudirohusodo or the networking hospitals where the study took place.

Table 2 presents the mean of MBL in EONS group between the referral patients and non referral patients. MBL levels in EONS samples from referral hospital has median $0.87 \mu \mathrm{g} / \mathrm{mL}$, SD $0.37 \mu \mathrm{g} / \mathrm{mL}$, and range of $0.17-1.70 \mu \mathrm{g} / \mathrm{mL}$. While in EONS samples that are not from referral hospital has median value of $0.89 \mu \mathrm{g} / \mathrm{mL}$, SD $0.24 \mu \mathrm{g} / \mathrm{mL}$, and range $0.23-1.06 \mu \mathrm{g} / \mathrm{mL}$. Statistical test results between both group shows no significant difference with $\mathrm{p}=0.930$.

Table 2. MBL mean value of EONS group in referral and non referral patients.

\begin{tabular}{lll}
\hline MBL serum $(\boldsymbol{\mu g} / \mathbf{m L})$ & Referral $(\mathbf{n}=\mathbf{2 2})$ & Non Referral $(\mathbf{n}=\mathbf{2 4})$ \\
\hline Mean & 0.88 & 0.79 \\
Median & 0.87 & 0.89 \\
SD & 0.37 & 0.24 \\
Range & $0.17-1.70$ & $0.23-1.06$ \\
Mann-Whitney Test & & $p=0.930$ \\
\hline
\end{tabular}

Table 3 shows the mean value of MBL on EONS group and not EONS group. MBL levels in EONS group have a median value of $0.88 \mathrm{mg} / \mathrm{mL}, \mathrm{SD} 0.31 \mathrm{mg} / \mathrm{mL}$, and range 0.17 to $1.70 \mathrm{mg} / \mathrm{mL}$. While, the not EONS group has median of $0.93 \mu \mathrm{g} / \mathrm{mL}$, SD $0.39 \mu \mathrm{g} / \mathrm{mL}$, and range $0.84-2.95$ $\mu \mathrm{g} / \mathrm{mL}$. Statistical results between both group shows significant difference with $p=0.000$.

Table 3. MBL mean on groups of EONS and not EONS.

\begin{tabular}{lll}
\hline MBL serum $(\boldsymbol{\mu g} / \mathbf{m L})$ & EONS $(\mathbf{n}=\mathbf{4 6})$ & Not EONS $(\mathbf{n}=\mathbf{4 6})$ \\
\hline Mean & 0.84 & 1.09 \\
Median & 0.88 & 0.93 \\
SD & 0.31 & 0.39 \\
Range & $0.17-1.70$ & $0.84-2.95$ \\
Mann-Whitney Test & & $p=0.000$ \\
\hline
\end{tabular}

Table 4 shows the sensitivity, specificity, positive predictive value, and negative predictive value from each cut off point of serum MBL levels. In the calculation, MBL levels of 0.93 has the biggest AUC with value of 0.630 ; with sensitivity of $76 \%$, specificity $56 \%$, positive predictive value $60 \%$, negative predictive value $67 \%$, and $p=0.031$.

Table 4. Sensitivity, specificity, positive predictive value, and negative predictive value of each cut off point of MBL serum levels.

\begin{tabular}{|c|c|c|c|c|c|c|}
\hline $\begin{array}{l}\text { MBL LEVELS } \\
(\mu \mathrm{g} / \mathrm{mL})\end{array}$ & Sensitivity (\%) & Specificity (\%) & NPP (\%) & NPN (\%) & AUC & $p$ \\
\hline 0.93 & 76 & 56 & 60 & 67 & 0.630 & 0.031 \\
\hline 0.94 & 78 & 43 & 58 & 66 & 0.609 & 0.072 \\
\hline 0.95 & 78 & 36 & 55 & 62 & 0.576 & 0.209 \\
\hline 0.96 & 78 & 32 & 53 & 60 & 0.554 & 0.369 \\
\hline 0.97 & 78 & 32 & 53 & 60 & 0.554 & 0.369 \\
\hline
\end{tabular}

NPP: Positive predictive value, NPN: Negative predictive value, AUC: Area under the Curve 
Table 5 presents the analyses result evaluation of cut off point of MBL 0.93 on the outcomes. Frequency of EONS in MBL group $0.93 \mu \mathrm{g} / \mathrm{mL}$ is as many as $76.1 \%$ and the frequency of not EONS in MBL group $>0.93 \mu \mathrm{g} / \mathrm{mL}$ is $50 \%$. Statistical analyses shows a significant difference between cut off point serum MBL levels $\leq 0.93 \mu \mathrm{g} / \mathrm{mL}$ and the incidence of EONS $(p=0.01)$.

Table 5. Analyses result evaluation of cut off point MBL 0.93 on the outcomes.

\begin{tabular}{lll}
\hline MBL & EONS n (\%) & Not EONS n (\%) \\
\hline$\leq 0.93 \mu \mathrm{g} / \mathrm{mL}$ & $35(76.1)$ & $23(50)$ \\
$>0.93 \mu \mathrm{g} / \mathrm{mL}$ & $11(23.9)$ & $23(50)$ \\
Total & $46(100)$ & $46(100)$ \\
\hline
\end{tabular}

Pearson Chi-Square $\mathrm{p}=0.01 \mathrm{COR}=3.182(\mathrm{IK} 95 \% 1.306-7.752)$

\section{Discussion}

This is a cross sectional study to assess the serum MBL levels on EONS in newborn. The MBL levels in EONS samples from referral hospital has median of $0.87 \mu \mathrm{g} / \mathrm{mL}$, SD $0.37 \mu \mathrm{g} / \mathrm{mL}$, and range $0.17-1.70 \mu \mathrm{g} / \mathrm{mL}$. While in EONS samples that are not from referral hospital median value is $0.89 \mu \mathrm{g} / \mathrm{mL}$, SD $0.24 \mu \mathrm{g} / \mathrm{mL}$, and range $0.23-1.06 \mu \mathrm{g} / \mathrm{mL}$. A statistical test result between both groups shows no significant difference with $p=0.930$. This shows that MBL response in infants from referral hospital and infants that are not from referral hospital are different, in this case, the different MBL response is due to the presence of the trigger (exposure to infection) and due to the genetic variation. Both referral and non-referral patients are exposed to infection which lead to sepsis within the first 72 hours, with the same diagnosis criteria. Either referral or not referral infants are from the South Sulawesi province; therefore, there are no significant genetic differences between both groups.

Total of samples is 92 , which 54 samples (58.7\%) are from the maternal fever, and 38 samples $(41.3 \%)$ are from the non maternal fever. Samples from the mother with leucocytosis are $19(20.7 \%)$, while the not leucocytosis are $73(79.3 \%)$. Samples from the mother with PROM are 37 (40.2\%) and not PROM is $55(59.8 \%)$. There are 79 samples $(85.9 \%)$ with green meconial amniotic fluid, and 13 samples (14.1) with clear amniotic fluid. There are $42(45.7 \%)$ male and 50 $(54.3 \%)$ female, and the statistical test shows there are no significant difference between sex distrmothertion on the incidence of EONS with $p=0.67$. This means that sex distrmothertion in EONS and not EONS group is homogenic; therefore, no further analyses are required to assess the influence of sex on EONS incidence. Similar results were also demonstrated by BJ Stoll et al., that sex of the infant were not associated with the risk of early-onset sepsis. ${ }^{11}$

Each risk factor of distrmothertion, which are mother's temperature, leukocyte levels, PROM, and amniotic fluid color in EONS and not EONS group shows there are no significant difference between each risk factor on the incidence of EONS.
MBL levels on EONS group has median of $0.88 \mu \mathrm{g} / \mathrm{mL}$, SD $0.31 \mu \mathrm{g} / \mathrm{mL}$, and range $0.17-1.70 \mu \mathrm{g} / \mathrm{mL}$. While, on the not EONS group has median of $0.93 \mu \mathrm{g} / \mathrm{mL}$, SD $0.39 \mu \mathrm{g} / \mathrm{mL}$, and range $0.84-2.95 \mu \mathrm{g} / \mathrm{mL}$. Statistical test result between both groups shows significant difference with $p=0.000$. This result is similar with a study performed by Ozkan N. et al. who obtained median value of MBL levels in sepsis infants was significantly lower than those without sepsis, with $p<0.05$. $^{7}$ Study by Frakking et al., demonstrated that EONS infants with low MBL levels is more frequent than the EONS infants with normal MBL levels. ${ }^{12}$

This indicates that although the mother risk factor is present, either fevers, leucocytosis, PROM, etc, but when the infant shows no symptoms of infection, then the antibiotic treatment is not required. ${ }^{13}$

Although there is statistically significant difference, but the median and range of MBL levels in both groups are very close, indicating that this difference is not too far apart and there is overlapping of MBL levels in samples of EONS and not EONS. This means, there are EONS samples with high MBL levels that are included within range of not EONS group, and there are also not EONS samples with low MBL levels within EONS group; therefore, it reducing the relationship significance between $\mathrm{MBL}$ levels on the incidence of EONS.

This finding also supported by ROC analyses result in order to find a cutoff point to differentiate the EONS and not EONS, where it is found that cut off point for MBL levels in both group is between 0.84 to 1.7 . Based on the accuracy calculation of MBL levels on each cut off point, it is found that cut off point MBL of 0.93 has the biggest Area Under Curve (AUC) of 0.630 ; with sensitivity of $76 \%$, specificity $56 \%$, positive predictive value $60 \%$, negative predictive value $67 \%$, and value $p=0.031$.

This result shows that the cutoff point cannot be used as a diagnostic value for EONS. The result is different from the previous studies by Mohammed et al. who found the cutoff point of MBL $0.7 \mu \mathrm{g} / \mathrm{mL}(p<0.05)$, Schlapbach et al, found the cutoff point of $0.3 \mu \mathrm{g} / \mathrm{mL}(p=0.028)$, Frakking et al., with cut off point of $0.2 \mu \mathrm{g} / \mathrm{mL}(p<0.01){ }^{9}, 12,14$ This differences because of in this study all sample are a term babies, whereas in study by Mohamed et al. Schlapbach et al. and Frakking et al. the subjects were both a term and preterm babies. From this study, it is known that the MBL levels of a term baby is higher than in preterm baby, thus, a term baby's immune response is better than preterm baby.

The EONS frequency in group of MBL $\leq 0.93 \mu \mathrm{g} / \mathrm{mL}$ is $76.1 \%$ and the frequency of not EONS in group of MBL $>0.93 \mu \mathrm{g} / \mathrm{mL}$ is $50 \%$. Statistical analyses shows significant difference between cut off point of MBL serum $\leq 0.93 \mu \mathrm{g} / \mathrm{mL}$ and the incidence of EONS $(p=0.01)$. The EONS frequency is 3 times higher in MBL serum $\leq 0.93$ $\mu \mathrm{g} / \mathrm{mL}$ compared with MBL serum $>0.93$.

This result support the previous studies which stated that infant with low serum MBL are more likely to have neonatal sepsis. ${ }^{9,}$ 12, 14 Therefore, in newborn, non-specific immunity 
plays important role in eliminating pathogens, thus newborn with low MBL levels are prone to sepsis.

This result is very important to be used as determine of EONS incidence and in effort to improve the newborn management (based current evidence). This result becomes the basis to perform routine serum MBL test in newborn with highly suspected sepsis. Therefore, if a baby is suspected to have EONS with low MBL (MBL $\leq 0.93 \mu \mathrm{g} / \mathrm{mL})$ prompt treatment must be started immediately. Early treatment is beneficial in suppressing sepsis induced morbidity and mortality rate. The limitation of this study is in its methods which is a cross sectional study, we couldn't establish a cause and the relationship effect because both of the risk and effect data are collected at the same time. In this case it is not known whether low MBL lead to EONS. The strength of this study is the data used is a primary data and samples are from the big hospital which becomes a referral center in East Indonesia, especially in South Sulawesi.

We conclude that the serum MBL levels in newborn with EONS is lower than newborn without EONS. The EONS frequency in newborn with $\mathrm{MBL} \leq 0.93 \mu \mathrm{g} / \mathrm{mL}$ is 3 times higher compared to newborn with MBL serum $>0.93 \mu \mathrm{g} / \mathrm{mL}$. We recommend performing a cohort study in the future to see the role of serum MBL in predicting the neonatal sepsis, and also to conduct further study about comparison of serum MBL measured serially/periodically to detect any changes in MBL serum right after delivery and the baby develop into sepsis. Thus it is hoped to help explaining the immune system pathomechanism in newborn and also to perform a study on the association between MBL levels, MBL genetic polymorphism with the incidence of neonatal sepsis in South Sulawesi. We also recommend performing a routine serum MBL examination in highly suspected sepsis infants, to perform prompt treatment in those who have low MBL serum to prevent disease progression and to re-evaluate criteria for EONS diagnosis.

\section{References}

[1] Alamsyah, Effek. Epidemiological analysis of neonatal maternal health efforts in Indonesia in achieving the target of millenium development goals (mdgs) 2015. Buletin Perinasia. 2010; (1).

[2] WHO. Neonatal sepsis - A major killer to be tackled in communities. www.who.int/maternal_child_adolescent. 2010.

[3] Karanda, I Made. Incidence and Factors Associated With Mortality Of Neonatal Sepsis. Pediatrica Indonesiana. 2011; Vol.51.
[4] Gomella, T.L., Cunningham, M.D., Eyal, F.G., Zenk, K.E. Infectious Diseases. In Neonatology: Management, Procedures, On-Call Problems, Diseases, and Drugs. $5^{\text {th }}$ ed. McGraw-Hill Companies. 2004; h.434-81.

[5] Zaghouani H, Hoeman CM. Neonatal immunity : Faulty T Helper and Shortcoming Of Dendritic Cells. Trends Immunol; 2009; 30: 585-591.

[6] Thiel S. Complement Activating Soluble Pattern Recognotion Molecules With Collagen-Like Regions, Mannan-Binding Lectin, Ficolins And Associated Proteins. Mol Immunol. 2007; 44: 3875-3888.

[7] Özkan, H., Köksal, N., Çetinkaya, M, Kılıç Ş., Çelebi S, Oral, B., et al. Serum mannose-binding lectin (MBL) gene polymorphism and low MBL levels are associated with neonatal sepsis and pneumonia. Journal of Perinatology. 2012; $32,210-217$.

[8] Benedetti, F.D., Auritia, C., Prenciped, G., Inglesec, R., Azzarie, C., Ronchettia, M.P., Tozzib, A., Segantia, R., and Orzalesia, M. Role of Mannose-Binding Lectin in Nosocomial Sepsis in Critically Ill Neonates (Online). Human Immunology. Itali. 2010; Vol 71: 1084-8.

[9] Schlapbach L.J., Mattmann M., Thiel S., Boillat C., Otth M., Nelle M., et al. Differential Role of the Lectin Pathway of Complement Activation in Susceptibility to Neonatal Sepsis. 2010. 1058-4838/2010/5102-0006.

[10] Hibberd, Martin L., Summerfield, John A. Levin, Michael. Variation in Mannose Binding Lectin (MBL) Gene and Susceptibility to sepsis. Departemen of Paediatrics and Medicine, Imperial College Faculty of Medicine. London. Springe. 2001; 4 (3): 201-207.

[11] Barbara J. Stoll, Nellie Hansen, Avroy A. Fanaroff, Linda L. Wright, Waldemar A. Carlo,Richard A. Ehrenkranz, et.al.. Changes in Pathogens Causing Early-Onset Sepsis in VeryLow-Birth-Weight Infants. The New England Journal of Medicines. 2002; 347:240-247.

[12] Frakking, F.N.J., Brouwer, N., Eijkelenburg, N.K.A. van, Merkus, M.P., Kuijpers, T.W., Offringa, M., and Dolman, K.M. Low Mannose-binding Lectin (MBL) Levels in Neonates with Pneumonia and Sepsis. British Society for Immunology, Clinical and Experimental Immunology. 2007; 150: 255-62.

[13] Health Ministry of Indonesia. Management of Sepsis Neonatorum. www.buk.depkes.go.id. 2007.

[14] Mohamed, W. A. Wahab and Saeed, M. A. Mannose-Binding Lectin Serum Levels in Neonatal Sepsis and Septic Shock. Journal of Maternal-Fetal and Neonatal Medicine. 2012; 25 (4): 411-414. 